\title{
MSCT Lung Perfusion Imaging Based on Multi-stage Registration
}

\author{
Helen Hong ${ }^{1}$ and Jeongjin Lee ${ }^{2, *}$ \\ ${ }^{1}$ School of Electrical Engineering and Computer Science \\ BK21: Information Technology, Seoul National University \\ hlhong@cse.snu.ac.kr \\ ${ }^{2}$ School of Electrical Engineering and Computer Science, Seoul National University, \\ San 56-1 Shinlim 9-dong Kwanak-gu, Seoul 151-742, Korea \\ jjlee@cglab.snu.ac.kr
}

\begin{abstract}
We propose a novel subtraction-based method for visualizing segmental and subsegmental pulmonary embolism. For the registration of a pair of CT angiography, a proper geometrical transformation is found through the following steps: First, point-based rough registration is performed for correcting the gross translational mismatch. The center of inertia (COI), apex and hilar point of each unilateral lung are proposed as the reference point. Second, the initial alignment is refined by iterative surface registration. Third, thin-plate spline warping is used to accurately align inner region of lung parenchyma. Finally, enhanced vessels are visualized by subtracting registered pre-contrast images from post-contrast images. To facilitate visualization of parenchymal enhancement, color-coded mapping and image fusion is used. Our method has been successfully applied to four pairs of CT angiography.
\end{abstract}

\section{Introduction}

Currently, computed tomography (CT) has become increasingly important in the diagnosis of pulmonary embolism because of the advent of multi-detector row CT scanners providing high spatial and excellent contrast resolution [1-3]. In CT angiography (CTA) images, thrombi are generally recognized as dark regions within enhanced pulmonary arteries. Thus the basis of pulmonary embolism assessment on CT images is the direct visualization of contrast material within the pulmonary arteries. However, it provides only limited information on perfusion defects since lung parenchymal attenuation changes as a result of the injection of contrast material are too faint to be identified on segmental and subsegmental vessels. If lung perfusion can be well visualized, CT may provide more accurate information on pulmonary embolism.

Several methods have been suggested for visualizing perfusion defects in CTA [4]. Mastuni et al. [5] proposed a fully automatic detection method based on segmentation of pulmonary vessels to limit the search space and analysis of several 3D features inside segmented vessel volume. However, several false positive occurs due to flow

\footnotetext{
* Corresponding author.
} 
void and soft tissue between adjacent vessels. Zhou et al. [6] developed a CAD system for detection of pulmonary embolism in CTA images. An adaptive 3D pixel clustering method was developed based on Baysian estimation and ExpectationMaximization (EM) analysis to segment vessels. Then the vessel tree was reconstructed by tracking the vessel and its branches in 3D space based on their geometric characteristics such as the tracked vessel direction and skeleton. Pichon et al. [7] proposed a method to highlight potential pulmonary embolism in a 3D representation of the pulmonary arterial tree. At first, lung vessels are segmented using mathematical morphology. The density values inside the vessels are then used to color the outside of a SSD of the vessel tree. However, pulmonary vessels exhibit a wider distribution of CT values from slice to slice. Thus it is difficult to visualize vessel structures in 3D volume using segmentation-based approach for the pulmonary embolism diagnosis since vessels cannot be accurately segmented and continuously tracked if they are largely or totally clotted by pulmonary embolism. Herzog et al. [89] proposed an image post-processing algorithm for visualization of parenchymal attenuation in chest CT angiography, which divided into five steps: lung contour segmentation, vessel cutting, adaptive filtering, color-coding and overlay with the original images. However, the method has a limitation in the direct visualization of emboli by CT angiography alone. Chung et al. [10] evaluated the value of CT perfusion image obtained by $2 \mathrm{D}$ mutual information-based registration and subtraction for the detection of pulmonary embolism. However, they evaluated their method using a porcine model under the limited conditions. The $2 \mathrm{D}$ registration has a limitation to accurately align three-dimensional anatomy. In addition, the processing time is about 40 seconds for single slice registration. Thus, it is difficult to be useful and acceptable technique for clinical applications in diagnosis of pulmonary embolism.

Current approaches still need more progress in computational efficiency and accuracy for detecting attenuation changes of pulmonary vessels in CTA. In this paper, we propose a novel subtraction-based method for accurately imaging perfusion defects and efficiently detecting segmental and sub-segmental pulmonary embolism in chest CTA images. For the registration of a pair of CTA, a proper geometrical transformation is found through the following steps: First, point-based rough registration is performed for correcting the gross translational mismatch. The center of inertia (COI), apex and hilar point of each unilateral lung are proposed as the reference point. Second, the rough alignment is refined by iterative surface registration. For fast and robust convergence of the distance measure to the optimal value, a $3 \mathrm{D}$ distance map is generated by the narrow-band distance propagation. Third, thin-plate spline warping is used to accurately align inner region of lung parenchyma. Finally, enhanced vessels are visualized by subtracting pre-contrast images from registered post-contrast images. To facilitate visualization of parenchymal enhancement, color-coded mapping and image fusion is used.

The organization of the paper is as follows. In Section 2, we discuss how to correct the gross translational mismatch. Then we propose a narrow-band distance propagation to generate a 3D distance map and a distance measure to find an exact geometrical relationship in pre- and post-contrast images of CTA. Finally, nonrigid registration using thin-plate spline warping is described to align deformable and distorted area within lung parenchyma. In Section 3, experimental results show how 
our registration method accurately and rapidly aligns the lungs. This paper is concluded with brief discussion of the results in Section 4.

\section{Lung Perfusion Imaging}

Fig. 1 shows the pipeline of our method for lung perfusion imaging in pre- and postcontrast images of chest CTA. In order to extract the precise lung region borders, pulmonary vessels and main airway, we apply the automatic segmentation method of Yim et al. [11] to our experimental datasets. Since our method is applied to the diagnosis of pulmonary embolism, we assume that each CT scan is almost acquired at the maximal inspiration and the dataset includes the thorax from the trachea to below the diaphragm.

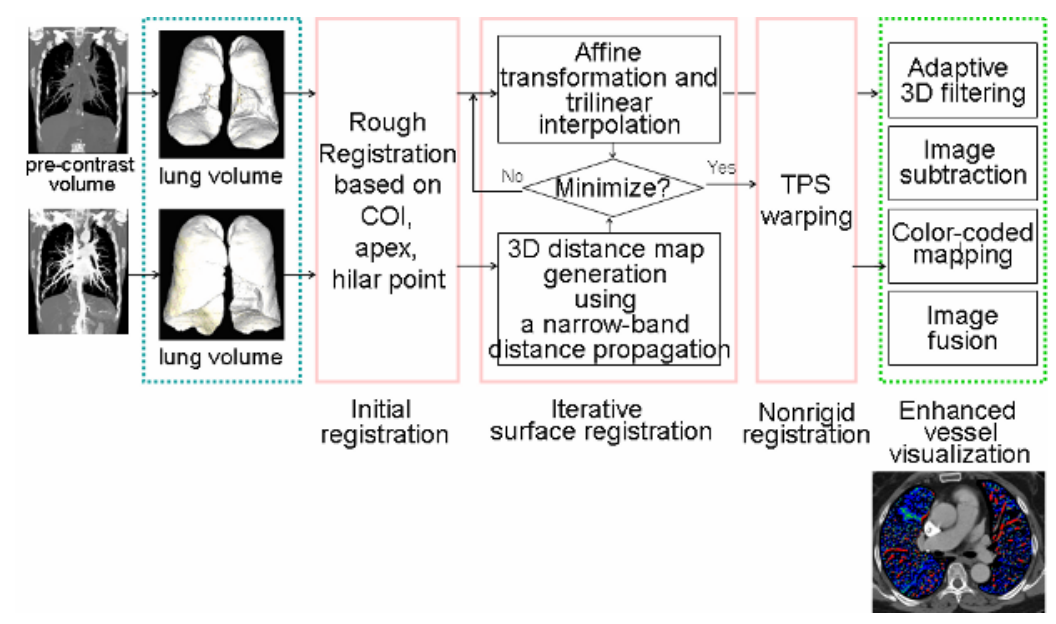

Fig. 1. The pipeline of proposed method for visualization of pulmonary embolism

\subsection{Point-Based Rough Registration}

Although pre- and post-contrast images of chest CT angiography are acquired at the maximal inspiration, the position of lung boundaries between pre- and post-contrast images can be quite different according to the patient's unexpected respiration and small movement. For the efficient registration of such images, an initial gross correction method is usually applied. Several landmark-based registration techniques have been used for the initial gross correction. To achieve the initial alignment of lung boundaries, these landmark-based registrations require the detection of landmarks and point-to-point registration of corresponding landmarks. These processes much degrade the performance of the whole process.

To minimize the computation time and maximize the effectiveness of initial registration, we propose a point-based rough registration using hilar point and evaluate our method with center of inertia and apex. As shown in Fig. 2(c), hilar point is where the outermost upper lobe vein crosses the basal artery on its way to the left 


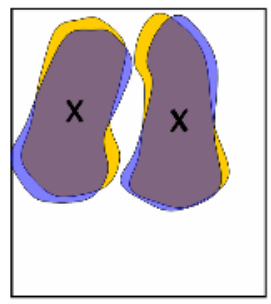

(a)

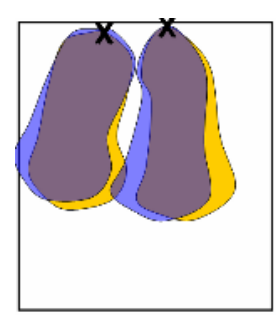

(b)

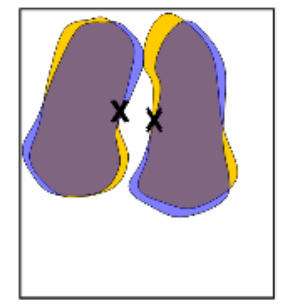

(c)

Fig. 2. The effect of point-based rough registration as an initial alignment (a) COI-based registration (b) apex-based registration (c) hilar point-based registration

atrium. The initial registration of two volumes is accomplished by aligning the COI, apex and hilar point, respectively.

The processing time of point-based rough registration is dramatically reduced since it does not require any anatomical landmark detection. In addition, our method leads to robust convergence to the optimal value since the search space is limited near the lungs.

\subsection{Iterative Refinement Using Surface Registration}

In a surface registration algorithm, the calculation of the distance from a surface boundary to a certain point can be done using a preprocessed distance map based on chamfer matching. Chamfer matching reduces the generation time of a distance map by an approximated distance transformation compared to a Euclidean distance transformation. However, the computation time of distance is still expensive by the two-step distance transformation of forward and backward masks. In particular, when the initial alignment almost corrects the gross translational mismatch, the generation of a 3D distance map of whole volume is unnecessary. From this observation, we propose the narrow-band distance propagation for the efficient generation of a 3D distance map.

To generate a 3D distance map, we approximate the global distance computation with repeated propagation of local distances within a small neighborhood. To approximate Euclidean distances, we consider 26-neighbor relations for 1-distance propagation as shown in Eq. (1). The distance value tells how far it is apart from a surface boundary point. The narrow-band distance propagation is applied to surface boundary points only in the contrast volume. We can generate a 3D distance map very fast since pixels are propagated only in the direction of increasing distances to the maximum neighborhood.

$$
D P(i)=\min \left(\min _{j \in 26-\text { neighbors }(i)}(D P(j)+1), D P(i)\right) .
$$

The distance measure in Eq. (2) is used to determine the degree of resemblance of lung boundaries of mask and contrast volume. The average of absolute distance difference, $A A D D$, reaches the minimum when lung boundary points of mask and contrast volumes are aligned correctly. Since the search space of our distance measure 
is limited to the surrounding lung boundaries, the Powell's method is sufficient for evaluating $A A D D$ instead of using a more powerful optimization algorithm.

$$
A A D D=\frac{1}{N_{\text {mask }}} \sum_{i=0}^{N_{\text {mask }}-1}\left|D_{\text {contrast }}(i)-D_{\text {mask }}(i)\right|
$$

where $D_{\text {mask }}(i)$ and $D_{\text {contrast }}(i)$ is the distance value of mask volume and the distance value of the $3 \mathrm{D}$ distance map of contrast volume, respectively. We assume that $D_{\text {mask }}(i)$ are all set to $0 . N_{C}$ is the total number of surface boundary points in mask volume.

\subsection{Non-rigid Registration Using Thin-Plate Spline Warping}

Affine transformation in iterative surface registration is insufficient for accurate modeling of inner lung parenchyma since the lung volumes move in a non-linear way influenced by a combination of body movement, heart beats, and respiration. Thus we use a thin-plate spline warping using 10 control points of vascular structure in each unilateral lung. Our method leads to a non-linear volumetric warping for aligning inner region of lung parenchyma and detecting pulmonary embolism accurately.

Thin-plate splines can be defined as a linear combination of radial basis functions as shown in Eq. (3). A transformation between two volumes can be defined by three separate thin-plate splines.

$$
t(x, y, z)=a_{1}+a_{2} x+a_{3} y+a_{4} z+\sum_{i=1}^{N} b_{i} \theta\left(\left|\phi_{i}-(x, y, z)\right|\right), \theta(s)=|s|,
$$

where $\phi_{i}$ is ith control point. The coefficient $a_{i}$ characterizes the linear part of the transformation and the coefficient $b_{i}$ characterizes the non-linear part of the transformation.

\subsection{Enhanced Vessel Visualization}

A traditional approach for visualizing enhanced vessels after registration is to subtract registered pre-contrast volume from post-contrast volume. However, it is difficult to easily recognize perfusion defects using a traditional subtraction technique when lung parenchymal changes as a result of the injection of contrast material are too small. After subtraction, we apply color-coded mapping to only lung parenchyma and image fusion with original image.

To facilitate visualization of parenchymal enhancement, the subtraction image is mapped onto a spectral color scale, which is interactively controlled by modifying center and width of a spectral color. Then the resulting color-coded parenchymal images are overlaid onto the corresponding slice of contrast volume. For overlaying, all non-parenchymal pixels are replaced by the original pixels of the respective slice position and displayed in the usual CT gray-scale presentation. 


\section{Experimental Results}

All our implementation and test were performed on an Intel Pentium IV PC containing $3.4 \mathrm{GHz}$ and $2.0 \mathrm{~GB}$ of main memory. Our method has been applied to four clinical datasets with pulmonary embolism, as described in Table 1, obtained from Siemens Sensation 16-channel multidetector row CT scanner. The image size of all experimental datasets is $512 \times 512$. The pre- and post-contrast images of chest CT angiography are acquired under the same image conditions excepting the injection of contrast material.

Table 1. Image conditions of experimental datasets

\begin{tabular}{cccccc}
\hline & Subject \# & Image size & Slice number & Pixel size & Slice thickness \\
\hline \hline \multirow{2}{*}{1} & Pre-contrast & $512 \times 512$ & 258 & $0.60 \times 0.60$ & 1.5 \\
& Post-contrast & $512 \times 512$ & 258 & $0.60 \times 0.60$ & 1.5 \\
\hline \multirow{2}{*}{2} & Pre-contrast & $512 \times 512$ & 175 & $0.61 \times 0.61$ & 1.5 \\
& Post-contrast & $512 \times 512$ & 175 & $0.61 \times 0.61$ & 1.5 \\
\hline \multirow{2}{*}{3} & Pre-contrast & $512 \times 512$ & 221 & $0.69 \times 0.69$ & 1.5 \\
& Post-contrast & $512 \times 512$ & 221 & $0.69 \times 0.69$ & 1.5 \\
\hline \multirow{2}{*}{4} & Pre-contrast & $512 \times 512$ & 214 & $0.59 \times 0.59$ & 1.5 \\
& Post-contrast & $512 \times 512$ & 214 & $0.59 \times 0.59$ & 1.5 \\
\hline
\end{tabular}

The performance of our method is evaluated with the aspects of visual inspection, accuracy and total processing time. Fig. 3 shows the results of color-coded mapping and image fusion on original image. Segmental and subsegmental emboli are detected predominantly in the upper lobe of right and left lungs as shown in Fig. 3. We can easily recognize the occlusion of the corresponding segmental and subsegmental arteries as color-coded mapping and fusion.

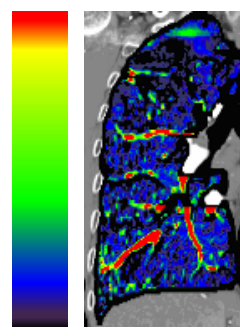

(a)

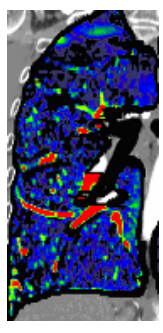

(b)

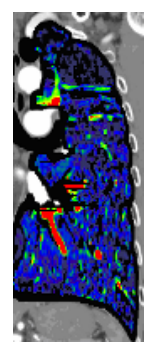

(c)

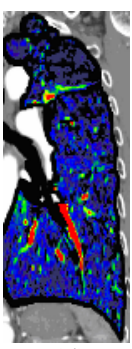

(d)

Fig. 3. The results of color-coded mapping and image fusion in subject 1

Fig. 4 shows how the error, the average of root-mean squared error of corresponding control points, is reduced by our rough registration. The average RMS error reduction of COI- and hilar point-based registration is $0.32 \mathrm{~mm}$ and $0.27 \mathrm{~mm}$, respectively. However, $0.72 \mathrm{~mm}$ is increased in the average RMS error using apexbased rough registration. 


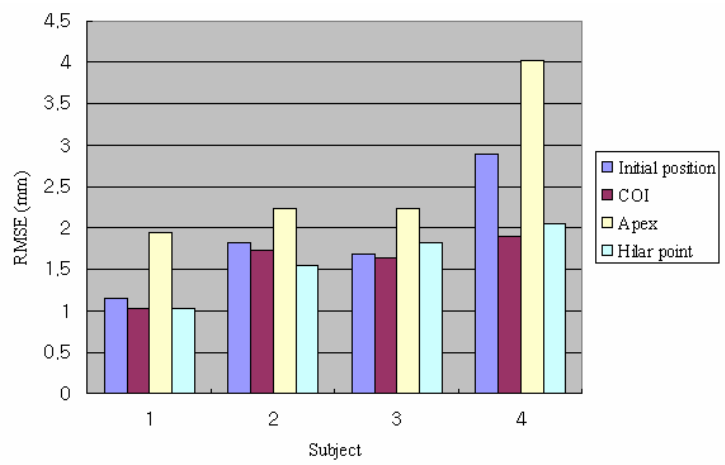

Fig. 4. The accuracy evaluation of corresponding points after rough registration

Fig. 5 shows how the error, the average of absolute distance difference (AADD), is reduced by our rough registration and subsequent iterative surface registration. The COI-, apex- and hilar point-based registration is used as rough registration shown in Fig. 5(a), (b) and (c), respectively. Since positional difference is almost aligned by our rough registration, iterative surface registration rapidly converge to the optimal position. In almost clinical datasets, the AADD errors are less than 0.6 voxels on optimal solution.

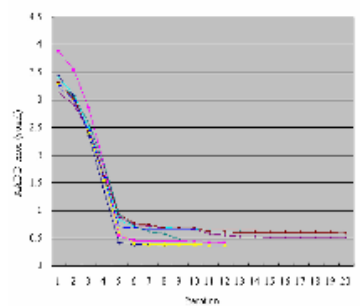

(a)

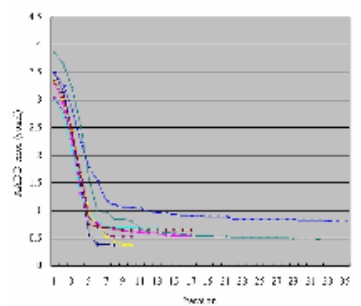

(b)

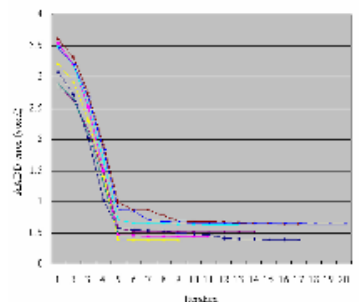

(c)

Fig. 5. The accuracy evaluation of corresponding lung boundaries using AADD error per iteration

Fig. 6 shows the results of our method (Method 3) of four patients in comparison with COI-based rough registration (Method 1) and apex-based rough registration (Method 2). The average of RMS errors of Method 1 and Method 3 as shown in Fig. 6 (a) and (c) are all $1.12 \mathrm{~mm}$. In contrary to them, the average of RMS error of Method 2 as shown in Fig. 6 (b) is $1.25 \mathrm{~mm}$. In conclusion, the average of RMS error is relatively small when COI- or hilar point-based registration is used as the initial alignment. The total processing time is summarized in Table 2 where the execution time is measured for registration. For four subjects, it takes less than 10 minutes. 


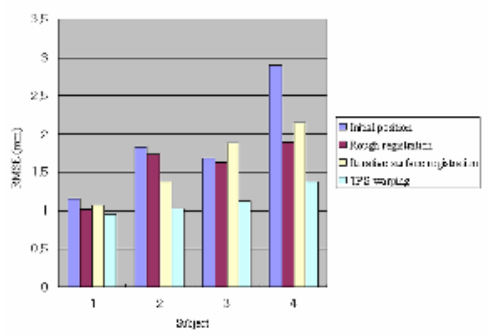

(a)

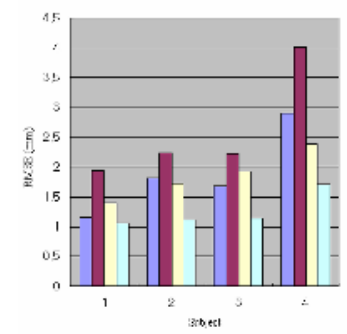

(b)

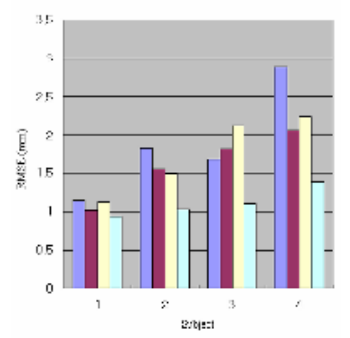

(c)

Fig. 6. The accuracy evaluation of corresponding lung boundaries using AADD error per subject

Table 2. The comparison of processing time for registration

\begin{tabular}{|c|c|c|c|c|c|}
\hline \multicolumn{2}{|c|}{ Subject \# } & $\begin{array}{c}\text { Iterative } \\
\text { surface } \\
\text { registration }\end{array}$ & TPS warping & $\begin{array}{c}\text { Enhanced } \\
\text { vessel } \\
\text { visualization }\end{array}$ & $\begin{array}{c}\text { Total } \\
\text { processing } \\
\text { time }\end{array}$ \\
\hline \multirow{4}{*}{1} & Method 1 & 80.656 & 532.906 & 63.011 & 676.573 \\
\cline { 2 - 6 } & Method 2 & 80.716 & 533.387 & 62.970 & 677.073 \\
\cline { 2 - 6 } & Method 3 & 80.576 & 533.557 & 62.921 & 677.054 \\
\hline \multirow{3}{*}{2} & Method 1 & 43.833 & 238.002 & 39.077 & 320.912 \\
\cline { 2 - 6 } & Method 2 & 43.453 & 237.832 & 39.116 & 320.401 \\
\cline { 2 - 6 } & Method 3 & 43.954 & 239.935 & 39.076 & 322.965 \\
\hline \multirow{3}{*}{3} & Method 1 & 75.088 & 374.889 & 51.194 & 501.171 \\
\cline { 2 - 6 } & Method 2 & 74.467 & 373.758 & 51.103 & 499.328 \\
\cline { 2 - 6 } & Method 3 & 74.847 & 375.911 & 51.123 & 501.881 \\
\hline \multirow{4}{*}{4} & Method 1 & 71.993 & 342.663 & 49.311 & 463.967 \\
\cline { 2 - 6 } & Method 2 & 70.591 & 340.620 & 49.261 & 460.472 \\
\cline { 2 - 6 } & Method 3 & 72.174 & 341.030 & 49.231 & 462.435 \\
\hline \multirow{3}{*}{$\begin{array}{c}\text { Avera } \\
\text { ge }\end{array}$} & Method 1 & 67.893 & 372.115 & 50.648 & 490.656 \\
\cline { 2 - 6 } & Method 2 & 67.307 & 371.399 & 50.613 & 489.319 \\
\cline { 2 - 6 } & Method 3 & 67.888 & 372.608 & 50.588 & 491.084 \\
\hline
\end{tabular}

\section{Conclusion}

We have developed a new subtraction-based method for visualizing perfusion defects in pre- and post-contrast images of CT angiography. Using the rough registration, the initial gross correction of the lungs can be done much fast and effective without detecting any anatomical landmarks. In the subsequent iterative surface registration, our distance measure using a 3D distance map generated by the narrow-band distance propagation allows rapid and robust convergence to the optimal value. Nonrigid registration using thin-plate spline warping can exactly aligns inner region of lung parenchyma. Our enhanced vessel visualization makes the recognition of attenuation variations within lung parenchyma easily. Four pairs of pre- and post-contrast images of CT angiography have been used for the performance evaluation with the aspects of visual inspection, accuracy and processing time. In visual inspection, we can easily recognize the occlusion of the corresponding segmental and subsegmental arteries. The registration error of our method is less than $1.12 \mathrm{~mm}$. All our registration process 
is finished within 10 minutes. Accurate and fast result of our method can be successfully used to visualize pulmonary perfusion for the diagnosis of pulmonary embolism.

\section{References}

1. Schoepf, U.J., Costello, P., CT angiography for diagnosis of pulmonary embolism: state of the art, Radiology, Vol. 230 (2004) 329-337.

2. Patel, S., Kazerooni, E.A., Cascade, P.N., Pulmonary embolism: optimization of small pulmonary artery visualization at multi-detector row CT, Radiology, Vol. 227 (2003) 455460.

3. Schoepf, U.J., Holzknecht, N., Helmberger, T.K. et al, Subsegmental pulmonary emboli: improved detection with thin-collimation multi-detector row spiral CT, Radiology, Vol. 222 (2002) 483-490.

4. Ko, J.P., Naidich, D.P., Computer-aided diagnosis and the evaluation of lung disease, Journal of Thoraic Imaging, Vol. 19, No. 3 (2004) 136-155.

5. Masutani, Y., MacMahon, H., Doi, K., Computerized detection of pulmonary embolism in spiral CT angiography based on volumetric image analysis, IEEE Trans. on Medical Imaging, Vol. 21, No. 12 (2002) 1517-1523.

6. Zhou C, Hadjiisk LM, Sahiner B. et al., Computerized detection of pulmonary embolism in 3D computed tomographic images: vessel tracking and segmentation technique, Proc. of SPIE Medical Imaging, Vol. 5032 (2003) 1613-1620.

7. Pinchon E, Novak CL, Naidich DP, A novel method for pulmonary emboli visualization from high resolution CT images, Proc. of SPIE Medical Imaging, Vol. 5061 (2004).

8. Herzog, P., Wildberger, J.E., Niethammer, $M$ et al., CT perfusion imaging of the lung in pulmonary embolism, Acad Radiol, Vol. 10 (2003) 1132-1146.

9. Wildberger, J.E., Schoepf, U.J., Mahnken, A.H., et al., Approaches to CT perfusion imaging in pulmonary embolism, Roentgenology (2005) 64-73.

10. Chung, M.J., Goo, J.M., Im, J.G., et al., CT perfusion image of the lung : value in the detection of pulmonary embolism in a porcine model, Investigative Radiology, Vol. 39, No. 10 (2004) 633-640.

11. Yim, Y., Hong, H., Shin, Y.G., Hybrid lung segmentation in chest CT images for computer-aided diagnosis, Proc. of HEALTHCOM 2005 (2005). 